\title{
Effet du mode de présentation du même fourrage (foin ou herbe pâturée) sur les caractéristiques de fromages de Saint-Nectaire affinés
}

\author{
JB Coulon ${ }^{1}$, I Verdier ${ }^{2}$, P Pradel ${ }^{3}$ \\ ${ }^{1}$ Laboratoire adaptation des herbivores aux milieux, Inra Theix, 63122 Saint-Genès-Champanelle ; \\ ${ }^{2}$ Laboratoire de recherches fromagères, Inra, 36, rue de Salers, 15000 Aurillac, France; \\ ${ }^{3}$ Domaine de la Borie, Inra, 15190 Marcenat, France
}

(Reçu le 9 avril 1996 ; accepté le 17 juin 1996)

\begin{abstract}
Résumé - Seize vaches multipares en milieu de lactation ont été utilisées dans un essai en carré latin $2 \times 2$. Pendant chaque période de 3 semaines, les animaux ont reçu une ration à base de fourrage de prairie naturelle offerte soit sous forme de foin récolté au premier cycle, soit sous forme d'herbe verte, issue de la même parcelle et pâturée en deuxième cycle. Le lait de ces vaches a été transformé en fromages de Saint-Nectaire, dans des conditions technologiques de fabrication contrôlées et identiques d'une fabrication à l'autre. La production laitière a été supérieure avec l'herbe pâturée $(+1,8 \mathrm{~kg} / \mathrm{j}, p<0,01)$. La teneur en urée du lait a été supérieure avec le foin, et la teneur en calcium légèrement inférieure. Les autres constituants chimiques du lait, son $\mathrm{pH}$ et ses caractéristiques rhéologiques ont été voisins dans les deux traitements. La plupart des caractéristiques physiques et sensorielles des fromages ont été semblables dans les deux traitements. Les fromages ont cependant été plus fermes et plus acides lorsque le fourrage était offert sous forme de foin.
\end{abstract}

fromage / alimentation / caractéristique sensorielle / vache laitière

Summary - Effect of forage type (hay or grazing) on milk cheesemaking ability. Sixteen multiparous dairy cows in mid-lactation were used in an experiment with a $2 \times 2$ Latin-square design. During each 3-week period, the cows were offered a diet composed of native mountain grassland given as hay (first cut) or grazed (second cut of the same plot). The milk yielded by these cows was used in the manufacture of Saint-Nectaire cheeses, under controlled technology manufacturing conditions which were identical from one production to another. Milk yield was higher at pasture $(+1.8 \mathrm{~kg} /$ day, $\mathrm{P}<0.01)$. Urea content was higher with hay $(+5.6 \mathrm{mg} / 100 \mathrm{~mL}, \mathrm{P}<0.01)$, and calcium content was slightly lower $(-0.05 \mathrm{~g} / \mathrm{kg}, \mathrm{P}<0.05)$. Other chemical contents (fat, protein, casein), $\mathrm{pH}$ and renneting properties of milk were similar in both treatments, as most of the chemical (dry matter, fat in dry matter, $\mathrm{pH}$ ) and sensory characteristics (taste, odour, colour) of the cheeses. However, cheeses were firmer and more acid when forage was given as hay.

cheese / feeding / sensory characteristic / dairy cow 


\section{INTRODUCTION}

La nature des aliments, et en particulier celle des fourrages, est souvent invoquée pour expliquer certaines variations des caractéristiques des produits laitiers et en particulier des fromages affinés (Keen et Wilson, 1993 ; Linden et Chamba, 1994 ; Martin et Coulon, 1995b). Ainsi, de nombreuses observations empiriques attribuent des vertus spécifiques aux fourrages consommés en vert (Urbach, 1990). Si quelques études ont été récemment entreprises pour mettre en évidence l'effet du mode de conservation sur la composition chimique du lait et les caractéristiques du fromage (Verdier et al, 1995), il existe cependant très peu de travaux expérimentaux comparant le fourrage vert et le foin correspondant, permettant de séparer l'effet propre de la fenaison des autres facteurs de variation d'origine technologique, physiologique ou alimentaire.

On sait cependant que la fenaison entraîne des modifications sensibles de la composition chimique du fourrage (Demarquilly, 1987), dont certaines ont des conséquences sur la composition du lait (composition des matières grasses, teneurs en carotène...) qui peuvent se répercuter sur les caractéristiques du fromage.

L'objectif de cet article a été d'analyser l'effet, sur les caractéristiques du lait et du fromage affiné, d'une ration à base de fourrage de prairie naturelle selon qu'elle était offerte sous forme de foin récolté au premier cycle ou d'herbe verte issue de la même parcelle et pâturée en deuxième cycle.

\section{MATÉRIEL ET MÉTHODES}

\section{Schéma expérimental et alimentation}

Seize vaches multipares Holstein $(n=10)$ ou Montbéliardes $(n=6)$ en pleine lactation (stade moyen au début de l'essai : 150 jours) ont été utilisées dans un essai en inversion avec deux périodes de 3 semaines. Entre le 15 mai et le 27 juin, elles ont été conduites ensemble, au pâtu- râge. À partir du 27 juin, deux lots de huit vaches ont été constitués sur la base de la race, du variant génétique de la caséine $\kappa$, de la production et de la composition chimique du lait au cours des 3 semaines précédentes. Deux parcelles identiques de 3 hectares ont été récoltées en foin (séché en grange) fin mai pour la première et fin juin pour la seconde. Ces parcelles étaient composées pour environ $90 \%$ de graminées, essentiellement de paturin commun $(32 \%)$, de raygrass anglais $(9 \%)$, de dactyle $(9 \%)$, de brome mou $(8 \%)$ et de vulpin $(6 \%)$.

La première parcelle a été pâturée au cours de la première période expérimentale, et la seconde au cours de la seconde période expérimentale. Sur ces deux parcelles, le pâturage a été conduit au fil (changé deux fois par jour). Au cours d'une première période de 3 semaines, le premier lot est resté au pâturage, sur la première parcelle, tandis que le second lot a reçu, à l'étable, le foin récolté fin mai, offert à volonté. Le 18 juillet, les traitements ont été inversés. Le 8 août, tous les animaux ont été rassemblés au pâturage. Dans les deux traitements, les animaux ont été complémentés à l'aide d'un concentré de type production $\left(1,04 \mathrm{UFL} \mathrm{L}^{1} / \mathrm{kg}\right.$ de matière sèche [MS], $122 \mathrm{~g}$ de $\mathrm{PDI}^{2} / \mathrm{kg}$ de MS), selon les recommandations Inra (1988), à raison de $1 \mathrm{~kg}$ par tranche de $2 \mathrm{~kg}$ de lait au dessus de $20 \mathrm{~kg} / \mathrm{j}$ à l'étable et de $22 \mathrm{~kg} / \mathrm{j}$ au pâturage. Durant tout l'essai, les vaches ont reçu $200 \mathrm{~g} / \mathrm{j}$ d'un même complément minéral ( $8 \mathrm{P}, 8 \mathrm{Ca}, 10 \mathrm{Mg})$.

\section{Mesures}

Sur les deux parcelles utilisées, la hauteur d'herbe a été mesurée, à l'aide d'un herbomètre, une fois par semaine durant l'essai. La composition chimique (matière organique, matières azotées totales et cellulose brute) a èté déterminée chaque semaine de fabrication fromagère, et une caractérisation floristique a été réalisée selon la méthode des contacts spécifiques (Daguet et Poissonet, 1969) en trois endroits différents de la parcelle. Les quantités de foin ingérées ont été mesurées 5 jours par semaine et les quantités de concentré tous les jours. La composition chimique du foin a été déterminée

\footnotetext{
${ }^{1}$ Unité fourragère lait (Inra, 1988).

${ }^{2}$ Protéines digestibles dans l'intestin grêle (Inra, 1988).
} 
à la récolte. La digestibilité de la matière sèche et de la matière organique a été déterminée en utilisant des lots de six moutons durant des périodes de mesure de 7 jours après une période d'adaptation de 15 jours. Ces donnees ont permis de calculer la valeur nutritive du foin, selon les recommandations de I'Inra (Andrieu et Demarquilly, 1987). Les caractéristiques des fourrages sont précisées dans le tableau I. Les vaches ont été pesées la semaine précédant la mise en lot et au cours de chacune des dernières semaines des deux périodes expérimentales. La production laitière a êté mesurée individuellement tous les jours et les teneurs en matières grasses et en protéines 3 jours par semaine sur chacune des deux traites journalières. Une fois par semaine au cours de l'essai, le pH du lait a été mesuré, juste après la traite du soir, à une température de $20^{\circ} \mathrm{C}$.

Une fois par semaine, au cours de la dernière semaine de chacune des deux périodes, un échantillon individuel de lait a été prélevé à la traite du matin pour analyse de la teneur en caséines (Rowland, 1938) en protéines solubles, en urée : méthode colorimétrique au diméthyl amino benzaldéhyde, et en calcium (total et soluble) (méthode par émission de flamme IOS, 1987) du lait.

Deux jours par semaine au cours de la dernière semaine de chacune des deux périodes expérimentales, des fabrications fromagères de saintnectaire ont été réalisées, à partir du lait des vaches de chaque lot. Les caractéristiques rhéologiques des laits de mélange mis en fabrication ont été mesurées, à l'aide d'un appareil Formagraph, selon la méthode proposée par McMahon et Brown (1982). La flore totale du lait (norme Afnor 1992), sa numération cellulaire (Somacount, Bentley), sa teneur en spores butyriques (Cerf et Bergère, 1968) et en bactéries coliformes (Leifson, 1935) ont été mesurées sur chacun des lots de lait, pour contrôler leur qualité sanitaire. Les valeurs observées pour ces différentes variables ont été constamment basses. Tous les laits ont donc été mis en fabrication.

Les fabrications ont été réalisées en mini-fromagerie expérimentale équipée de deux cuves d'une contenance maximale de $40 \mathrm{~L}$, permettant chacune de fabriquer deux fromages. Les fromages de Saint-Nectaire ont été fabriqués selon une technologie de type Saint-Nectaire laitier au lait cru à partir de laits de la traite de la veille au soir réfrigérés en tank et conservés à $4{ }^{\circ} \mathrm{C}$ jus- qu'à leur transformation. Cette technologie a été décrite en détail par Verdier et al (1995).

Sur un des fromages de chaque cuve, les analyses suivantes ont été réalisées. $\mathrm{Le} \mathrm{pH}$ a été mesuré à $20^{\circ} \mathrm{C}$ par introduction d'une électrode Ingold dans un broyat réalisé par mixage de $30 \mathrm{~g}$ de fromage. La teneur en matière sèche a été déterminée par dessiccation à $103{ }^{\circ} \mathrm{C}$ pendant 24 heures. Le dosage de la matière grasse des fromages a été réalisé par butyrométrie (norme FIL-IDF 152, 1991). L'indice de jaune de la pâte des fromages a été déterminé à l'aide d'un spectrophotomètre muni d'une sphère intégratrice, sur chacune des deux faces d'une tranche prélevée au centre des fromages.

Après 8 semaines d'affinage, les caractéristiques sensorielles des fromages ont été appréciées en deux temps de deux façons très differrentes.

Tous les fromages affinés ont été soumis à un jury de dégustation composé de huit personnes appartenant au laboratoire Inra d'Aurillac. La qualité des fromages a été appréciée selon les critères de la commission de contrôle du syndicat du SaintNectaire : les membres du jury ont attribué une note d'appréciation à l'aspect du fromage (/5), à l'aspect de la pâte (/5) et au goût (/10).

Par ailleurs, le second fromage de chaque cuve a été soumis à dégustation par un groupe d'analyse sensorielle composé de dix sujets bénévoles, entrainés à la reconnaissance des saveurs élémentaires (norme Afnor 1988) et familiarisés à l'analyse sensorielle et à ses outils. Ce jury a préalablement éte exercé à l'évaluation de diverses caractéristiques (aspect, odeur, goût, texture) de fromage de Saint-Nectaire du commerce. Un profil du produit a ensuite été établi par génération libre de descripteurs dont le nombre a été réduit avec l'accord du panel de dégustateurs (norme Afnor 1990; Barthélémy, 1990). Les sujets ont été entraînés à l'évaluation de l'intensité de chacun des descripteurs du profil définitif: La notation a été effectuée sur une échelle structurée de 0 à 10. Le profil comportait deux parties d'une quinzaine de descripteurs chacun, l'une concernant la description du produit en bouche, et l'autre l'aspect du fromage. Les sujets ont toujours commencé par la partie dégustation proprement dite : les échantillons de fromage écroûtés étaient codés et présentés simultanément à tous les dégustateurs dans un ordre aléatoire et différent pour chacun. La partie " aspect " du fromage a été évaluée sur un quart du fromage, codé de manière différente à la partie précédente. 
Tableau I. Composition et valeur nutritive des fourrages. Composition and nutritive value of forages.

Foin

Herbe

Période $1 \quad$ Période 2

\begin{tabular}{lccc}
\hline Âge moyen des repousses (j) & & 37 & 44 \\
Matière organique (MO) (\% MS) & 89,8 & 88,9 & 90,9 \\
Matière azotées totales $(\%$ MS) & 15,2 & 16,1 & 13,0 \\
Cellulose brute $(\%$ MS) & 29,4 & 23,9 & 27,3 \\
UFL ( $/ \mathrm{kg}$ de MS) & 0,71 & 0,90 & 0,80 \\
PDIN (g/kg de MS) & 92 & 104 & 84 \\
PDIE (g/kg de MS) & 86 & 103 & 91
\end{tabular}

\section{Analyse des données}

L'analyse des résultats a été réalisée à partir des données moyennes de la dernière semaine de chaque période expérimentale. Ces données ont été traitées par analyse de variance (SAS, 1987) en introduisant dans le modèle la nature de la ration, lanimal (ou le lot ayant servi à fabriquer les fromages) et la période expérimentale, ainsi que, pour l'analyse des caractéristiques du lait mesurées de manière individuelle, la race.

\section{RÉSULTATS ET DISCUSSION}

\section{Production, composition chimique et aptitude du lait à la coagulation (tableau II)}

Le foin utilisé dans cette étude, d'excellente qualité en raison de l'utilisation du séchage en grange, a été particulièrement bien ingéré : son ingestibilité calculée à partir des équations de Dulphy et al (1987) et Faverdin et al (1987), est de 0,95 UEL $3 / \mathrm{kg} \mathrm{MS}$, soit une valeur correspondant à celle attribuée à une herbe pâturée au 25 mai (Inra, 1988).

La production laitière a été en moyenne supérieure au pâturage $(+1,8 \mathrm{~kg} / \mathrm{j}, p<0,01)$. Cette différence ne s'est manifestée qu'au

\footnotetext{
${ }^{3}$ Unité d'encombrement lait (Inra, 1988).
}

cours de la première période $(+3,2 \mathrm{~kg} / \mathrm{j})$, vraisemblablement en raison du plus grand écart de valeur nutritive entre le foin et l'herbe pâturée en première période $(0,19 \mathrm{UFL} / \mathrm{kg} \mathrm{MS})$ qu'en seconde période $(0,09$ UFL/kg MS). En dehors du taux d'urée qui a été supérieur avec la ration à base de foin $(+5,6 \mathrm{mg} / 100 \mathrm{~mL}, p<0,01)$ et du taux de calcium total qui a été légèrement inférieur $(-0,05 \mathrm{~g} / \mathrm{kg}, p<0,05)$, la composition chimique du lait ainsi que son $\mathrm{pH}$ n'ont pas été significativement différents d'un traitement à l'autre. Cette absence d'écart de taux protéique et de $\mathrm{pH}$ entre les deux traitements explique que les caractéristiques rhéologiques, qui sont très dépendantes de ces deux variables (Remeuf et al, 1991 ; Martin et Coulon, 1995a), n'aient pas été différentes d'un type de fourrage à l'autre, comme nous l'avions observé en comparant, à l'auge, du foin et de l'herbe verte (Coulon, 1995).

La mise à l'herbe des lots 1 (passage période 2 à période post-expérimentale) et deux (passage période 1 à période 2) ne s'est pas accompagnée d'augmentation de la production laitière, contrairement à ce qui est observé lors de mises à l'herbe plus précoces, même lorsque les animaux sont correctement alimentés à l'étable (Coulon et al, 1988 ; Coulon, 1995), vraisemblablement en raison de la moins bonne qualité 
Tableau II. Caractéristiques du lait.

Milk characteristics.

\begin{tabular}{|c|c|c|c|c|}
\hline & Foin & $\begin{array}{c}\text { Herbe } \\
\text { pâturée }\end{array}$ & & $E T R^{1}$ \\
\hline Fourrages (kg MS/j) & 16,0 & & & \\
\hline Concentrés (kg MS/j) & 2,6 & 1,0 & & \\
\hline Lait $(\mathrm{kg} / \mathrm{j})$ & 17,9 & 19,7 & $\star \star$ & 1,0 \\
\hline Matières grasses $(\mathrm{g} / \mathrm{j})$ & 630 & 698 & ** & 43 \\
\hline Matières protéiques $(\mathrm{g} / \mathrm{j})$ & 606 & 550 & $\star \star \star$ & 42 \\
\hline Taux butyreux (g/kg) & 34,9 & 35,6 & NS & 1,6 \\
\hline Taux protéique $(\mathrm{g} / \mathrm{kg})$ & 30,4 & 30,8 & NS & 0,9 \\
\hline Taux de caséines $(\mathrm{g} / \mathrm{kg})$ & 25,3 & 25,5 & NS & 0,9 \\
\hline Caséines/protéines & 0,82 & 0,82 & NS & 0,01 \\
\hline Calcium total $(\mathrm{g} / \mathrm{kg})$ & 1,46 & 1,51 & * & 0,06 \\
\hline Calcium soluble $(\mathrm{g} / \mathrm{kg})$ & 0,34 & 0,37 & NS & 0,05 \\
\hline Urée $(\mathrm{mg} / 100 \mathrm{~mL})$ & 43,4 & 37,8 & $\star \star$ & 5,0 \\
\hline Protéines solubles $(\mathrm{g} / \mathrm{kg})$ & 5,5 & 5,5 & NS & 0,7 \\
\hline $\mathrm{pH}$ & 6,60 & 6,61 & NS & 0,05 \\
\hline \multicolumn{5}{|l|}{ Mesures à $\mathrm{pH}$ initial : } \\
\hline Temps de gélification (min) & 10,6 & 9,9 & NS & 1,0 \\
\hline Temps de raffermissement (min) & 4,7 & 4,4 & NS & 0,5 \\
\hline Fermeté du gel à 1 fois le temps de gélification $(\mathrm{mm})$ & 31,0 & 31,2 & NS & 2,4 \\
\hline \multicolumn{5}{|l|}{ Mesures à pH standardisé $(6,60)$ : } \\
\hline Temps de gélification (min) & 9,8 & 9,5 & NS & 0,9 \\
\hline Temps de raffermissement (min) & 4,9 & 4,5 & NS & 0,7 \\
\hline Fermeté du gel à 1 fois le temps de gélification $(\mathrm{mm})$ & 26,7 & 29,3 & NS & 3,2 \\
\hline
\end{tabular}

${ }^{1}$ Écart type résiduel. NS : non significatif, ${ }^{\star} p<0,05,{ }^{\star \star} p<0,01$.

${ }^{1}$ Residual standard deviation.

de l'herbe pâturée comparativement à une herbe de printemps, et peut-être aussi parce que les animaux avaient déjà été mis à l'herbe 1 mois auparavant. Ces deux mises à l'herbe se sont en revanche accompagnées d'une augmentation importante des taux butyreux $(+3 \mathrm{~g} / \mathrm{kg}$ en l'espace d'une semaine) et protéique $(+2 \mathrm{~g} / \mathrm{kg})$, mais de très courte durée ( 1 semaine).

\section{Caractéristiques des fromages affinés}

Les caractéristiques physico-chimiques du fromage (tableau III) ont été semblables pour les deux traitements. L'herbe pâturée a conduit à des fromages dont l'indice de luminosité a été plus faible $(p<0,1)$ et légèrement plus jaunes, mais de manière non significative. Cette absence d'écart est étonnante, compte tenu du fait que la teneur en carotènes des fourrages, responsable de la couleur jaune des fromages, chute très rapidement après la fauche (Demarquilly, 1987). II est possible que le séchage en grange ait permis de limiter cette chute, puisque le fourrage n'est resté que 32 heures au sol avant d'être ramassé.

Les écarts de goût, de texture et d'aspect entre les deux traitements sont faibles, mais toujours à l'avantage de la ration foin, condui- 
Tableau III. Caractéristiques des fromages affinés.

Ripened cheese characteristics.

\begin{tabular}{|c|c|c|c|c|}
\hline & Foin & $\begin{array}{c}\text { Herbe } \\
\text { pâturée }\end{array}$ & & $E T R^{1}$ \\
\hline Tauxbutyreux/taux protéique ${ }^{2}$ & 1,04 & 1,07 & + & 0,02 \\
\hline Poids (kg) & 1,80 & 1,79 & NS & 0,04 \\
\hline $\mathrm{pH}$ & 5,61 & 5,65 & NS & 0,07 \\
\hline Extrait sec & 55,5 & 56,1 & NS & 1,5 \\
\hline Gras & 27,8 & 28,1 & NS & 2,1 \\
\hline Gras/sec & 50,0 & 50,0 & NS & 3,0 \\
\hline Extrait sec dégraissé & 38,4 & 39,1 & NS & 1,6 \\
\hline \multicolumn{5}{|l|}{ Couleur } \\
\hline Luminosité & 84,1 & 83,3 & + & 0,9 \\
\hline Indice de jaune & 31,9 & 32,7 & NS & 1,0 \\
\hline Indice de rouge & 1,16 & 0,98 & * & 0,17 \\
\hline \multicolumn{5}{|l|}{ Dégustation } \\
\hline Goût (/10) & 6,5 & 6,2 & NS & 0,4 \\
\hline Aspect $(/ 5)$ & 3,9 & 3,7 & $\star \star$ & 0,1 \\
\hline Texture (/5) & 3,1 & 2,9 & NS & 0,4 \\
\hline Note totale (/20) & 13,5 & 12,8 & + & 0,8 \\
\hline \multicolumn{5}{|l|}{ Analyse sensorielle } \\
\hline Déformation & 1,2 & 1,7 & + & 0,3 \\
\hline Moisissure & 6,3 & 4,4 & * & 0,8 \\
\hline Aspect poisseux & 1,6 & 2,9 & NS & 1,2 \\
\hline Couleur de la pâte & 5,6 & 5,9 & NS & 0,4 \\
\hline Élasticité & 4,9 & 5,3 & NS & 0,7 \\
\hline Fermeté au toucher & 4,7 & 3,2 & $* \star$ & 0,4 \\
\hline Intensité de l'odeur & 5,4 & 5,2 & NS & 0,6 \\
\hline Texture collante & 3,7 & 3,6 & NS & 0,3 \\
\hline Texture fondante & 4,7 & 5,3 & NS & 0,7 \\
\hline Fermeté en bouche & 4,5 & 3,4 & + & 0,6 \\
\hline Intensité du goût & 5,6 & 6,0 & NS & 0,6 \\
\hline Persistance du goût & 6,0 & 5,9 & NS & 0,2 \\
\hline Acidité & 2,1 & 1,6 & + & 0,3 \\
\hline Amertume & 4,2 & 4,7 & NS & 0,5 \\
\hline
\end{tabular}

${ }^{1}$ Écart type résiduel. NS : non significatif, $+p<0,1,{ }^{*} p<0,05,{ }^{* *} p<0,01 .{ }^{2}$ Des laits mis en fabrication, après écrémage partiel.

${ }^{1}$ Residual standard deviation. ${ }^{2}$ Of milk used for cheese manufacture, after partial skimming. 
sant à une note globale de dégustation légèrement supérieure $(+0,7$ point, $p<0,1)$. Du point de vue sensoriel, aussi bien au toucher qu'en bouche, les fromages du traitement herbe sont apparus significativement moins fermes que ceux du traitement foin. Ces demiers ont par ailleurs présenté une acidité plus accentuée $(p<0,1)$. Globalement, les caractéristiques sensorielles des fromages ont cependant été voisines dans les deux traitements.

\section{CONCLUSION}

En définitive, les résultats de cette étude vont à l'encontre des observations empiriques qui conduisent souvent à attribuer au pâturage des modifications importantes des caractéristiques du fromage (Urbach, 1990 ; Martin et Coulon, 1995b). La seule modification sensible que nous ayons observée concerne la fermeté, plus grande avec le régime foin. Cette différence de fermeté peut être attribuée à une modification de nature des matières grasses du lait entre les deux régimes, les rations à base d'herbe conduisant généralement à une proportion plus importante d'acides gras à chaînes longues et insaturées (Decaen et Ghadaki, 1970 ; Coulon et al, 1988), présentant un point de fusion plus bas. Ce résultat est cependant contradictoire avec les observations faites sur du reblochon fermier, où les fromages d'été étaient apparus plus fermes que ceux d'hiver (Martin et Coulon, 1995b). Il est possible que les modifications chimiques liées à la dessiccation du fourrage (Demarquilly, 1987), et qui pourraient être à l'origine de modifications des caractéristiques organoleptiques des fromages, aient été limitées par l'utilisation du séchage en grange. II est aussi possible que les faibles différences que nous avons observées soient liées au type de pâturage utilisé, constitué de repousses dont les caractéristiques sont certainement assez représentatives d'un pâturage d'été, mais très différentes d'une herbe de prin- temps, dont on connaît, à la mise à l'herbe, les effets importants sur la composition du lait et son aptitude à la coagulation (Grandison et al, 1984).

Il est cependant plus vraisemblable que les modifications habituellement observées ne soient pas dues strictement à la méthode de conservation du fourrage, mais plutôt à d'autres changements concomitants au changement de régime (amélioration parfois considérable des apports nutritifs, conduisant à une augmentation du taux protéique et donc à la facilité d'égouttage des fromages, modification de la nature floristique du fourrage, modification de la complémentation en aliment concentré, évolution du stade de lactation des animaux, voire modification de la technologie mise en œuvre...), qui dans cet essai ont été maîtrisés (schéma expérimental en inversion, pas de modification de la composition chimique du lait, même nature floristique du fourrage).

\section{RÉFÉRENCES}

Afnor (1988) Méthodologie - Guide général pour la sélection, l'entrainement et le contrôle des sujets qualifiés, NF V 09-003

Afnor (1990) Méthodologie - Recherche de descripteurs pour l'élaboration d'un profil sensoriel, NF V 09-021

Afnor (1992) Microbiologie alimentaire - Méthode de routine pour le dénombrement des micro-organismes. Méthode par comptage des colonies obtenues à $30^{\circ} \mathrm{C}$, NF V 08-051

Andrieu J, Demarquilly C (1987) Valeur nutritive des fourrages : tables et prévision. Bull Tech CRZV Theix, INRA 70, 61-74

Barthélémy J (1990) Évaluation d'une grandeur sensorielle complexe : description quantifiée. In : Évaluation sensorielle, manuel méthodologique, Technique et documentation Lavoisier, Paris, 145-162

Cerf O, Bergère JL (1968) La numération des spores de clostridium et son application au lait et aux produits laitiers. Numération des différents groupes de Clostridium. Lait 48, 501-519

Coulon JB (1995) Efiets respectifs du changement de l'alimentation et de l'environnement lors de la mise à l'herbe sur la composition chimique et l'aptitude à la coagulation du lait. Ann Zootech, 44, 153-160

Coulon JB, D'Hour P, Petit M (1988) Influence of transition feeding pattern on milk production at the turnout of cows to pasture. Livest Prod Sci 20, 119-134 
Daguet P, Poissonet J (1969) Analyse phytologique des prairies. Applications agronomiques. CNRS-CEPE Montpellier, document 48, $67 \mathrm{pp}$

Decaen C, Ghadaki MB (1970) Variation de la sécrétion des acides gras des matières grasses du lait de vache à la mise à l'herbe et au cours des six premières semaines d'exploitation du fourrage vert. Ann Zootech 19, 399-411

Demarquilly C (1987) La fenaison : évolution de la plante au champ entre la fauche et la révolte. Perte d'eau, métabolisme, modification de la composition morphologique et chimique. In : Les fourrages secs : récolte, traitement, utilisation (C Demarquilly, ed), Inra, Paris, 23-46

Dulphy JP, Faverdin P, Micol D, Bocquier F (1987) Révision du système des unités d'encombrement (UE). Bull Tech CRZV Theix Inra 70, 35-48

Faverdin P, Hoden A, Coulon JB (1987) Recommandations alimentaires pour les vaches laitières. Bull Tech CRZV Theix Inra 70, 133-152

FIL-IDF (1991) Laits et produits laitiers : détermination de la teneur en matières grasses. Guide de directives générales appliquées aux méthodes butyrométriques. Norme 152

Grandison AS, Ford GD, Owen AJ, Millard D (1984) Chemical composition and coagulating properties of renneted Friesian milk during the transition from winter rations to spring grazing. J Dairy Res $51,69-78$

Inra (1988) Tables de l'alimentation des bovins, ovins et caprins. Inra, Paris, 197 pp

International Organization for Standardization (1987) Dried milk. Determination of sodium and potassium contents. Flame emission spectrophotometric method. (ISO/DIS 8070)

Keen AR, Wilson RD (1993) Pasture feeding. A contribution of additionnal flavour nuances to milkfat and meat flavour. In: Milkfat flavour forum, summary of proceedings. New Zealand Dairy Research Institute, Palmerston North, New Zealand, 24-31

Leifson $E$ (1935) New culture media based on sodium desoxycholate for the isolation of intestinal pathogenes and for the enumeration of colon bacilli in milk and water. J Pathol Bacteriol 40, 581-599

Linden G, Chamba JF (1994) La typicité des fromages : une réalité, un objectif. Sci Aliments 14, 573-580

Martin B, Coulon JB (1995a) Facteurs de production du lait et caractéristiques des fromages. I. Influence des facteurs de production sur l'aptitude à la coagulation des laits de troupeaux. Lait 75, 61-80

Martín B, Coulon JB (1995b) Facteurs de production du lait et caractéristiques des fromages. II. Influence des caractéristiques des laits de troupeaux et des pratiques fromagères sur les caractéristiques du reblochon de Savoie fermier. Lait 75, 133-149

McMahon DJ, Brown RJ (1982) Evaluation of Formagraph for comparing rennet solutions. J Dairy Sci65, 1639-1642

Remeuf F, Cossin V, Dervin C, Lenoir J, Tomassone R (1991) Relations entre les paramètres physico-chimiques des laits et leur aptitude fromagère. Lait 71 , 397-421

Rowland SJ (1938) The determination of the nitrogen distribution in milk. J Dairy Res 9, 42-46

SAS Institute Inc. (1987) SAS User's guide : statistics. SAS Institute Inc, Cary, NC

Urbach G (1990) Effect of feed on flavour in dairy foods. J Dairy Sci 73, 3639-3650

Verdier I, Coulon JB, Pradel P, Berdagué JL (1995) Effect of forage type and cow breed on the characteristics of matured Saint-Nectaire cheeses. Lait, 523-533 